A

Type of $1^{\text {st }}$ Operation - Colonic Resection (\%)

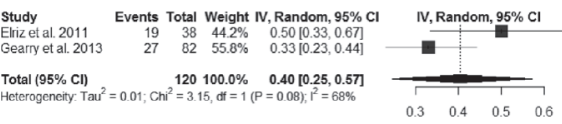

C

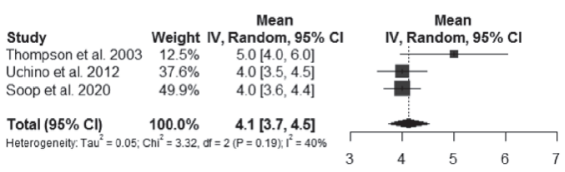

E Length of Small Bowel Remaining $(\leq 200 \mathrm{~cm})(\%)$

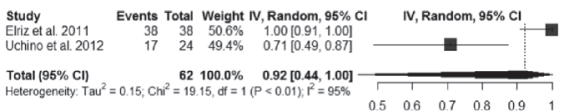

G

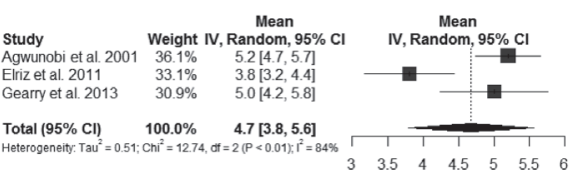

D

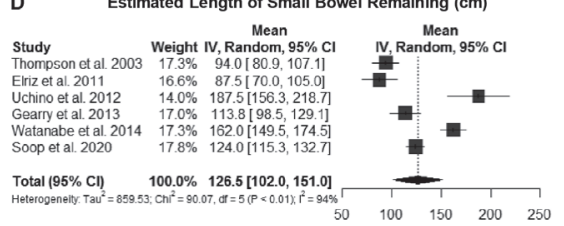

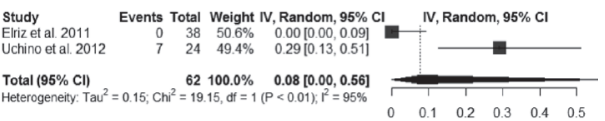

H Patients on whom Stricturoplasty was Performed (\%)

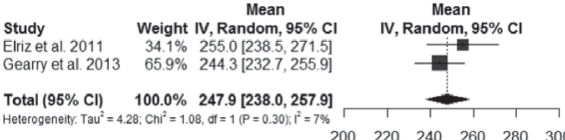
$\begin{array}{llllll}200 & 220 & 240 & 260 & 280 & 300\end{array}$

I Patients on whom Jejunostomy or lleostomy was performed (\%)

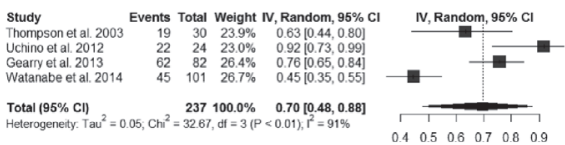

K Patients with a Left Colon Remnant (\%)

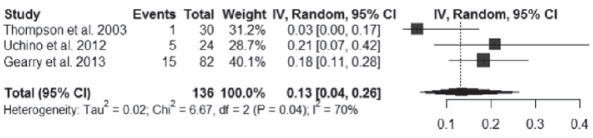

J Patients on whom Colostomy was performed (\%)

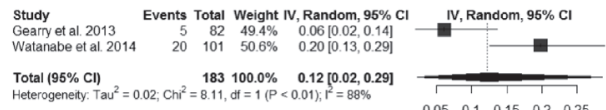

$\mathrm{L} \quad$ Patients with Partial or Total Preservation of Colon (\%)
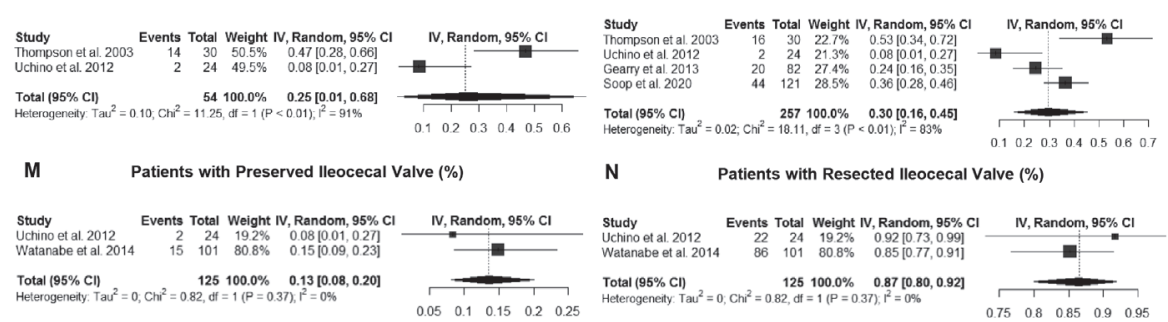

Abstract PM0-18 Figure 1 Meta-Analysis of Surgical Factors associated with IF

of smokers was $35.0 \%$. The mean duration of $\mathrm{CD}$ diagnosis before IF and initial surgery were $38.2 \mathrm{yrs} \pm 10.7$ and 6.8 yrs \pm 1.1 respectively. From meta-analysis, the mean number of small intestinal resections per patient were 4.7 (95\% CI: 3.8-5.6, $\left.\mathrm{P}<0.01, I^{2}=84 \%\right)$ and the mean estimated length of small bowel remaining was $126.5 \mathrm{~cm}$ (95\% CI: 102.0-151.0, $\mathrm{P}<0.01, I^{2}=94 \%$ ) (Figure 1). The mean estimated original small intestine length was $247.7 \mathrm{~cm}$ and the mean proportion of ileocecal valve resections were $87.0 \%$ (95\% CI: $80.0 \%$ 92.0\%, P=0.37, $I^{2}=0 \%$ ) (Figure 1). A colon remnant was present in $30.0 \%$ (95\% CI: $16.0 \%-45.0 \%, \mathrm{P}<0.01, I^{2}=83 \%$ ) and the majority of patients had a jejunostomy or ileostomy (70.0\%, 95\% CI: 48.0\%-88.0\%, P<0.01, $\left.I^{2}=91 \%\right)$. Total length of small bowel resected was $170.0 \mathrm{~cm} \pm 63.8$, small bowel resection length per procedure was $77.5 \mathrm{~cm} \pm 52.8$, and there was no significant difference in small bowel resection length between elective $(79.3 \mathrm{~cm} \pm 65.9)$ and emergency procedures $(118.5 \mathrm{~cm} \pm 81.7)$.

Conclusions Surgical risk factors in combination with medical and demographic factors can predispose patients to IF. However, certain surgical factors including ileocecal valve resection, presence of an ileostomy or jejunostomy, and small bowel length $\leq 200 \mathrm{~cm}$ could be predictive of the development of IF.

\section{PMO-19 BARRIERS AND FACILITATORS TO CLINICAL ACTIVITY INDICES FOR IBD IN ROUTINE PRACTICE: A QUALITATIVE STUDY}

Violeta Razanskaite*, Bridget Young, Paula Williamson, Keith Bodger. University of Liverpool, Liverpool, UK

\subsection{6/gutjnl-2021-BSG.158}

Introduction A recent national audit of biological therapies in the UK suggested that the recording of a disease activity index (Harvey-Bradshaw Index or Simple Colitis Activity index) was uncommon in routine settings. ${ }^{1}$ Our aims were to explore the barriers and facilitators to the use of clinical instruments in hospital-based inflammatory bowel disease (IBD) outpatient services, and the reasons for lack of standardisation in outcome assessment by practitioners.

Methods We performed structured observations of real-life consultations conducted with 102 patients by 24 clinicians (14 
doctors and 10 specialist nurses) at six hospitals across the North West region of England, followed by 24 qualitative semi-structured interviews with observed IBD practitioners. Qualitative data analysis was iterative and drew on thematic approaches to describe observed and self-reported practices, views and preferences.

Results During observed consultations, most clinicians performed personalised assessments of symptoms instead of using a standardised clinical index, and expressed preferences for individualised patient-centred evaluations of health status as opposed to a standardised approach. Practitioners captured indices in only $15 \%$ of observed consultations and stated that standardised tools were collected primarily for secondary uses of data and seen as an administrative burden with little relevance to clinical context. The perceived barriers to routine use of indices were 1) lack of application in individual decision making due to weak psychometric properties and poor specificity for inflammation, and 2) practical issues relating to constraints of time and lack of suitable IT infrastructure. Most commonly described facilitators were capture of patient reported outcomes instead of clinician-reported indices, userfriendly data entry tools, administrative support, training and regulatory oversight. Many clinicians, particularly doctors, remained sceptical about the benefits of using standardised assessment tools over clinical judgment.

Conclusions There are significant technical and human barriers to standardisation of clinical assessments for IBD in routine settings. These findings have several implications for efforts to implement clinical instruments in routine practice. Education and training, evidence-based guidance and better IT capabilities are needed in order to improve the capture of standardised information in busy clinical settings. Direct capture of patient reported outcomes is likely to provide a more feasible approach to capturing standardised outcomes as part of the care delivery process.

\section{REFERENCE}

1. Royal College of Physicians IBD Programme. National clinical audit of biological therapies. Annual report. 2016. https://www.rcplondon.ac.uk/projects/outputs/ national-clinical-audit-biological-therapies-annual-report-2016 (accessed 10 January 2021).

\section{\begin{tabular}{|l|l|l|l|}
\hline PMO-20 THE IBD BIOSIMILAR TO BIOSIMILAR SWITCHING \\
\hline
\end{tabular} STUDY (IBISS)}

1,2Clare Harris ${ }^{*},{ }^{1,2}$ Richard James Harris, ${ }^{1}$ David Young, 1,2Martin McDonnell, ${ }^{2}$ Bridget Clancy, ${ }^{3}$ Justin Harvey, ${ }^{2}$ Prof Susan Latter, ${ }^{1,2} \mathrm{JR}$ Fraser Cummings. ${ }^{1}$ University Hospital Southampton NHS Foundation Trust, Southampton, UK; ${ }^{2}$ University of Southampton, Southampton, UK; ${ }^{3}$ University of Stellenbosch, Stellenbosch, South Africa

\subsection{6/gutjnl-2021-BSG.159}

Introduction In 2013, the first biosimilar of infliximab was approved for use by the European Medicines Agency for the same indications as originator infliximab. The regulatory pathways involved compare the biosimilar with the originator only in controlled settings and not with other biosimilars. With the development of multiple biosimilars, patients may be asked to transition from one to another. There is a clear gap in the evidence with little data on switching between biosimilars. The aim of this study was to evaluate the clinical outcome of switching a cohort of IBD patients from CT-P13 to SB2 in a real-world setting, as well as explore the patient experience of having their medication switched.
Methods This was a prospective, phase IV interventional study at University Hospital Southampton. Patients treated with CTP13 (6/8 weekly regime at $5 \mathrm{mg} / \mathrm{kg})$ were approached and were switched to SB2. Demographics, disease history, validated disease activity scores (partial Mayo Score for UC and modified Harvey-Bradshaw Index for Crohn's), patient reported outcome measures (IBD Control PROM and PRO2) and laboratory measurements (full blood count, C-reactive protein, albumin and faecal calprotectin) were collected at each visit. Semi-structured qualitative interviews were also conducted to explore the patient experience of having their medication switched and were analysed using thematic analysis.

Results 133/158 patients approached participated in iBiSS with a mean disease duration of 9.2 years. The primary objective was clinical outcome at week 30/32. The mean mHBI and pMCS at week 0 vs week $30 / 32$ were 3.14 vs 2.9 and 1.53 vs $1.18(\mathrm{p}=0.77)$ respectively. The IBD Control-8 score for the whole cohort was 11.75 vs $13.19(\mathrm{p}=0.005)$ and the IBD Control-VAS was 75.24 vs $79.59(\mathrm{p}=0.57)$.

35 patients discontinued during the study (6 lost to followup, 12 due to adverse events, 4 withdrew consent, 13 for other reasons). There were 16 serious adverse events and no fatal adverse events.

Interviews were conducted on 26 participants. Six major themes were identified that reflected the patient experience. These included confidence through information, worry through information, trust in the clinical team, barriers to switching, motivators for switching and the fragility of their condition.

Conclusions The data presented here suggests there is no detrimental effect on the clinical outcomes of patients switched from one biosimilar of infliximab to another which is vital information to guide clinical practice.

\section{PMO-21 IMPACT OF COVID-19 ON INFLIXIMAB PRESCRIBING PRACTICES IN INFLAMMATORY BOWEL DISEASE}

${ }^{1}$ Stephanie Shields*, 'John Paul Seenan, ${ }^{2}$ Allan Dunlop, 'Jonathan Macdonald. 'Dept of Gastroenterology, Queen Elizabeth University Hospital, Glasgow, UK; ${ }^{2}$ Dept of Biochemistry, Queen Elizabeth University Hospital, Glasgow, UK

\subsection{6/gutjnl-2021-BSG.160}

Introduction The COVID-19 pandemic has impacted upon many aspects of clinical practice. As previously reported, use of biologic therapeutic drug monitoring (TDM) reduced markedly immediately after the UK wide lockdown of March 2020 , followed by a gradual increase in testing volume as lockdown measures were relaxed. ${ }^{1}$ Data from the Secure IBD registry suggested an increased risk of severe COVID with anti-TNF combination therapy (with a thiopurine) vs monotherapy. $^{2}$ The impact of the COVID-19 pandemic on biologic prescribing practices is largely unknown. The aim of this work was to assess the early effect of the COVID-19 pandemic on Scottish infliximab prescribing practices for individuals with IBD.

Methods IBD specific data was extracted from the Scottish biologic TDM database between $1 / 10 / 2019$ and $31 / 7 / 2020$ providing 5 months of data pre and post pandemic onset. Results were included for analysis if associated dosing and immunomodulator co-prescription data were available. Changes in concomitant immunomodulator (CI) prescription, dosing regimes and TDM strategy (proactive vs reactive) were reviewed. 